\title{
Bluetooth Technology: As a Home Appliances Controller via Android Mobile
}

\author{
Rajkumar Mistri \\ Asst. Professor, Dept. of ECE, RTCIT, Ranchi, India \\ Rahul Ranjan \\ Asst. Professor, Dept. of ECE, RTCIT, Ranchi, India \\ Shivani Singh \\ B.Tech Scholar, Dept. of ECE, RTCIT, Ranchi, India
}

\begin{abstract}
In today's era, the ease of life and simultaneously conservation of energy in most demanding thing. This should be the required contribution for every person for making a better world. In our proposed module we have designed a module which can control maximum four home appliances such as fan, cooler, AC, bulb etc. Via android mobile phone through Bluetooth technology. At this stage approximately every person have android mobile phone and in this paper our purpose and effort is to make maximum digitalization for home appliances. Our proposed module consist mainly two sections TX and RX. Our RX section of proposed module is very efficient and at the same time power consumption is very less. This module can be used efficiently at home, offices, schools, colleges and industries.
\end{abstract}

Keywords:- ATMEGA, TX, RX, NO, NC, COM, PCB, SPP, EDA, CAD, PROTEUS, DIP-TRACE, MCAD, EDA.

\section{INTRODUCTION}

Bluetooth Technology

Bluetooth is a radio technology that makes possible, transmitting signal over short distance between mobile phones, computers and other devices. It is a short range standard radio link which has unlicensed spectrum about $2.45 \mathrm{GHz}$ (ISM band 2400-2483MHz). Bluetooth Technology adopted Frequency Hopped Multiple access (FHMA) technology for power efficiency and low cost implementations. "A Bluetooth product, like headset or watch, contains a tiny computer chip with a Bluetooth radio and software that makes it easy to connect[1]. One need to pair Bluetooth devices to make communication between them over ad-hoc networks of shot range known as Pico-nets. In Pico-net network of Bluetooth devices, two and more devices can be connected. The connection is established dynamically and automatically on the basis of Master and Slaves, one device acts as Master and other acts as slave. Bluetooth technology not only connects our phone or computers or headset or speakers but also it can connect and control our home appliances such as fan coolers bulb, AC, door-locks, TV, Shoes, basketball toys and almost everything, we think to embed it based on microcontroller operated relays.

Relay

Relay is an electromagnetic device which is used to isolate two circuits electrically and connect them magnetically[2]. Relays can be used to interface between an electronic circuit to an high voltage electric circuit based on the principle of electromagnetic induction, for example a $230 \mathrm{~V} \mathrm{AC}$ main can be switched by a relay operated $5 \mathrm{~V}$ battery. In this way we can use relay circuits to drive our above said appliances efficiently according to our requirements. Input section of a Relay has a coil which generates magnetic field with implementation of a very small voltage from an electronic circuit, called the operating voltage. Commonly used relays with operating voltages are- $6 \mathrm{~V}, 9 \mathrm{~V}, 12 \mathrm{~V}, 24 \mathrm{~V}$. There are mainly three connectors in a basic relaynormally open (NO), normally closed (NC) and Common (COM). At no input state COM is connected to NC. At applied operating voltage coil gets energized and the COM make contact to NO. Depending on different change over contacts different relay configurations are available in the market such as-SPST, SPDT, DPDT etc. Here in our proposed RX part we have used SPDT relays.

Proteus Software

The Proteus Design Suite is an Electronic Design Automation (EDA) tool including schematic capture, simulation and PCB Layout modules[3]. This EDA Tool was developed in Yorkshire, England by Labcenter Electronics Ltd. Proteus supports Mixed mode SPICE Simulation, microcontroller simulation, Shape based 
auto-routing, 3D Board Visualisation and MCAD import/export. Proteus software runs on the Windows operating system and this supports English, French, Spanish and Chinese languages.

\section{Dipt-Race Software}

Dip-Trace is EDA/CAD Tool for creating schematic diagrams and printed circuit boards. Dip-Trace supports mainly five modules which are Schematic Capture Editor, PCB Layout Editor with built-in shape-based autorouter , 3D Preview \& Export, Component Editor and Pattern Editor[4]. Its schematic capture supports multilevel hierarchical schematics. This EDA tool supports schematic capture to PCB layout conversion.

\section{USED IC'S DESCRIPTION}

ATMEGA328P-PU

This is microcontroller based 28 pin ATMEGA328P-PU AVR series IC. It has 14 digital input/output pins out of which 6 pin can be used as a PWM output. This has also 6 Analog input pin. Operating frequency of this is $16 \mathrm{MHz}$. it has flash memory $32 \mathrm{kB}$, EPROM $2 \mathrm{~kb} \&$ RAM $2 \mathrm{~KB}$. It has also one UART, 4, 8-bit PWM output and 2, 16-bit PWM output. The pin configuration of ATMEGA328P-PU is described in Table-1.

Table1: Pin Detail of ATmega328

\begin{tabular}{|c|c|c|}
\hline PIN NO. & PIN NAME & MAPPED PIN NAME \\
\hline 1 & RESET & RESET \\
\hline 2 & RXD & RX/DIGITAL I/O 0 \\
\hline 3 & TXD & TX/ DIGITAL I/O 1 \\
\hline 4 & INT0 & DIGITAL I/O 2 \\
\hline 5 & INT1 & DIGITAL I/O 3 \\
\hline 6 & T0 & DIGITAL I/O 4 \\
\hline 7 & VDD & SUPPLY VOLTAGE \\
\hline 8 & GND & GROUND \\
\hline 9 & XTAL1 & CRYSTAL PIN 1 \\
\hline 10 & XTAL2 & CRYSTAL PIN 2 \\
\hline 11 & $\mathrm{~T} 1$ & DIGITAL I/O 5 \\
\hline 12 & AIN0 & DIGITAL I/O 6 \\
\hline 13 & AIN1 & DIGITAL I/O 7 \\
\hline 14 & ICP1 & DIGITAL I/O 8 \\
\hline 15 & $\mathrm{OC} 1 \mathrm{~A}$ & DIGITAL I/O 9 \\
\hline 16 & OC1B & DIGITAL I/O 10 \\
\hline 17 & MOSI & DIGITAL I/O 11 \\
\hline 18 & MISO & DIGITAL I/O 12 \\
\hline 19 & $\mathrm{SCK}$ & DIGITAL I/O 13 \\
\hline 20 & AVCC & SUPPLY VOLTAGE \\
\hline 21 & $\mathrm{AREF}$ & VREF \\
\hline 22 & GND & GROUND \\
\hline 23 & $\mathrm{ADC0}$ & ANALOG INPUT 0 \\
\hline 24 & $\mathrm{ADC1}$ & ANALOG INPUT 1 \\
\hline 25 & $\mathrm{ADC} 2$ & ANALOG INPUT 2 \\
\hline 26 & $\mathrm{ADC3}$ & ANALOG INPUT 3 \\
\hline 27 & ADC4 & ANALOG INPUT 4 \\
\hline 28 & ADC5 & ANALOG INPUT 5 \\
\hline
\end{tabular}

ULN2003A

ULN2003A is 16-pin based IC which contain seven NPN Darlington pair with common cathode clamp diode. In this IC collector current rating of a single Darlington pair is $500 \mathrm{~mA}$. Applications include relay

drivers, hammer drivers, lamp drivers, display drivers (LED and gas discharge), line drivers, and logic buffers[5].

pin description of IC is shown in Table-2. 
Table2: Pin Detail of ULN2003A

\begin{tabular}{|c|c|c|}
\hline PIN NO. & PIN NAME & PIN DESCRIPTION \\
\hline $1,2,3,4,5,6,7$ & INPUT $[0: 7]$ & Input Pins \\
\hline 8 & GND & Ground \\
\hline 9 & COM & High DC Supply Voltage \\
\hline $16,15,14,13,12,11,10$ & OUTPUT $[0: 7]$ & Output Pins \\
\hline
\end{tabular}

74HC573:

The 74HC573 has octal D-type transparent latches featuring separate D-type inputs for each latch and 3-state true outputs for bus-oriented applications [6]. This IC has 20 pin out of which two pins

Are used to control the data from input to output. Pin no. ' 1 ' which is denoted as OE which output enable signal. Since it is active low signal hence when $\mathrm{OE}=\mathrm{LOW}$ at this moment stored value of latches come to at its output otherwise outputs remains very high impedance. and similarly pin no-11 denoted as LE which indicate latch enable input since it is active high signal so inputs value (Din) will store in latches when LE=HIGH. The pin description of IC has briefly explained in Table-3.

Table3: Pin Detail of 74HC573

\begin{tabular}{|c|c|c|}
\hline PIN NO. & PIN NAME & PIN DESCRIPTION \\
\hline 1 & OE & 3-state output enable input (active LOW) \\
\hline $2,3,4,5,6,7,8,9$ & IN $[0: 7]$ & data inputs \\
\hline 10 & GND & ground \\
\hline 11 & LE & latch enable input (active HIGH) \\
\hline $19,18,17,16,14,13,12$ & OUT[0:7] & 3-state latch outputs \\
\hline 20 & VCC & supply voltage \\
\hline
\end{tabular}

LM7805:

LM7805 is a voltage regulator integrated circuit. It blocks the fluctuation of voltage at its output and keep voltage constant. This IC provide $+5 \mathrm{v}$ regulated output when DC value of $7 \mathrm{v}$ to $35 \mathrm{v}$ coming to its input.

BLUETOOTH MODULE (HC-06):

HC-06 module is a Bluetooth module which uses SPP(serial port protocol). This module also supports transparent wireless serial communication. This module has enhanced data rate of $3 \mathrm{Mbps}$ and also frequency band of $2.4 \mathrm{GHz}$. It has its nominal range of $10 \mathrm{~m}$. It has also channel bandwidth of $1 \mathrm{MHz}$ and also 16-bit CRC data protection.

Program for testing bluetooth module:

In order to test the module, Firstly Bluetooth module is connected to ARDUINO UNO board. RX pin of module is connected to digital I/O pin 3 of board and TX pin of module is connected to the digital I/O pin 2 of board and also GND as well as VDD pin of module is connected to ground and $+5 \mathrm{v}$ respectively of board and then program is uploaded to the board. After this when a character is sent by android via Bluetooth terminal after pairing the devices then same character will be displayed on serial monitor of ARDUINO software.

The program which is to be uploaded is given below

\#include $<$ SoftwareSerial.h $>$

SoftwareSerial mySerial(2,3); //RX-2, TX-3

char $\mathrm{c}=0$;

void $\operatorname{setup}()$

\{

Serial.begin(9600);

mySerial.begin(9600);

\}

void loop()

\{

while(1)

\{

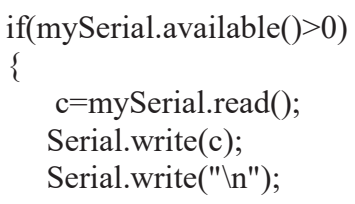


III. PCB PREPARATION FOR PROPOSED MODULE (RX PART)

Fig.1 Represents the PCB layout of our proposed receiver part. This has developed in Dip-Trace PCB Design Software.

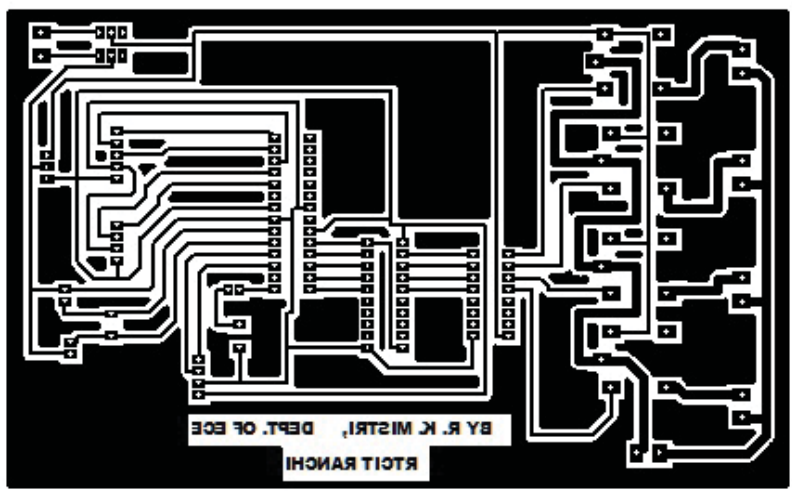

Fig.1: PCB layout of receiver part

Printing of layout structure of pcb to copper laminate:

In order to print layout structure of PCB to copper laminate firstly layout structure is printed on high glossy paper by laser printer with dark black ink. Then after copper is cleaned with thinner(acetone) and printed side of glossy paper is kept on copper laminate in such a manner that it can touch the copper side of laminate and also it is fixed by transparent tape. Then After a hot electric iron is kept on glossy paper and it is pressed continuously with movement of electric iron on whole glossy paper at least 3 minute so that flossy paper can stick with laminate. Then after laminate is dropped in normal water for a 3 minute. Further glossy paper is removed by hand with rubbing by keeping it in water. After doing so layout design will be printed on copper laminate, which will be looking like as shown in fig-1.

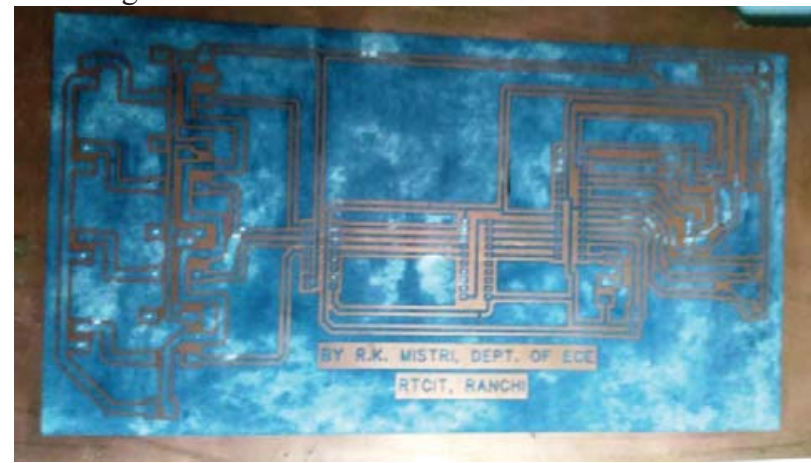

Fig.2: Printed layout structure of PCB on copper laminate

Etching of non-printed part of copper:

Etching is the process of removing non-printed part of copper from laminate. In order to remove non-printed part of copper from laminate, firstly an ferric chloride solution is prepared in platic type pot and then laminate is dipped into the solution and then solution is sacked well untill all non-printed copper remove from laminate. Now our aim is to remove ink which is removed by applying acetone. In this way our PCB become ready.

Masking And Protecting PCB:

To protect PCB, uv curable solder mask ink is applied to whole PCB and then mask is kept acordingly on pcb (which is printed on pvc sheet) and then system is kept on uv exposure system for 35 sec. Then mask ink become solid where uv light is incident on ink and otherwise area ink remains in liquid form that can be easily removed by cleaning $\mathrm{PCB}$ in $\mathrm{NaOH}$ solution. Now pcb become ready. So in order to put the component on PCB, firsty drilling is done by PCB Drilling Machineand then components or IC'S shockets are fitted on PCB accordingly and soldering is done.

\section{PROPOSED MODULE DESCRIPTION}


Our proposed model consists of two parts i.e. Transmitter part and Receiver part. Transmitter is nothing but an android mobile with installed Bluetooth terminal app. Our proposed receiver part contain Bluetooth module(HC-06), ATMEGA328P-PU AVR series microcontroller, 9V battery, LM7805 positive voltage regulator, 74HC573 octal d-type transparent latch, ULN2003A relay driver, DPDT 5v-relay and $16 \mathrm{MHz}$ crystal oscillator. Actually in our proposed module when a data is sent via android through Bluetooth terminal then sent data will remains available on serial port. At receiver part Bluetooth module support serial port protocol hence data available on serial is easily received by Bluetooth module when both are connected to each other. This received data is sent to microcontroller; according to received data microcontroller specify the work i.e. to on or off the relay. The schematic of our receiver part is shown in fig. 3 which has designed and simulated on PROTEUS software (EDA Tool).

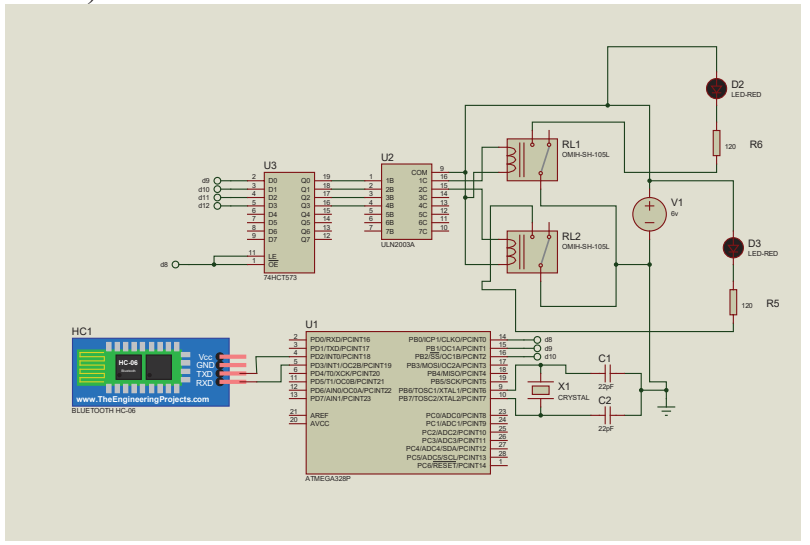

Fig.3: Schematic of RX part

Our proposed RX part can control two home appliances at a time because we have used only two relay in our hardware design instead of four. Our RX hardware part is shown in fig-4.

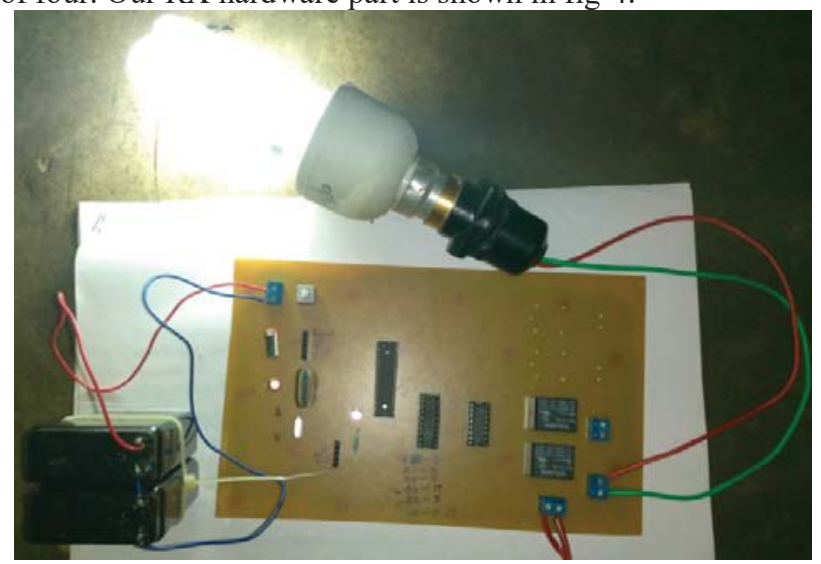

Fig.4: Proposed Hardware Design of RX part

When used Bluetooth module (HC-06) and Bluetooth terminal installed in android mobile are paired (connected) and information ' $\mathrm{A}$ ' is sent via terminal, then first relay change their state (either NC to NO or NO to NC). Similarly information ' $\mathrm{B}$ ' is used to control second relay.

\section{CONCLUSION \& FUTURE SCOPE}

Our proposed design is very useful in everywhere like offices, homes, schools, colleges, companies and many other places to control any kind electrical equipment. This system has very low power consumption and also has very low cost. As we know that Bluetooth has maximum $10 \mathrm{~m}$ range so it can only work in $0-10 \mathrm{~m}$ range. In future apps for android can be developed using apps development tools like (ANDROID STUDIO, ADK TOOL etc.) which may contain some button that can be used to control electric equipments at anywhere within $0-10 \mathrm{~m}$ range.

\section{REFRENCES}

[1] https://www.bluetooth.com/what-is-bluetooth-technology/bluetooth-technology-basics

[2] https://en.m.wikipedia.org/wiki/Relay

[3] https://en.m.wikipedia.org/wiki/Proteus_Design_Suite

[4] https://en.m.wikipedia.org/wiki/DipTrace

[5] www.ti.com/lit/ds/symlink/uln2003a.pdf

[6] http://www.nxp.com/documents/data_sheet/74HC_HCT573.pdf 